\title{
Efficacy, safety and effect on biomarkers of AZD9668 in cystic fibrosis
}

\author{
J. Stuart Elborn*, John Perrett", Kristina Forsman-Semb", \\ Joanna Marks-Konczalik", Kulasiri Gunawardena ${ }^{\#}$ and Neil Entwistle ${ }^{\#}$
}

ABSTRACT: The aim of this study was to evaluate the safety and effect on clinical outcomes and biomarkers of inflammation and tissue damage of the neutrophil elastase inhibitor AZD9668 (60 $\mathrm{mg}$ twice daily orally for 4 weeks) in cystic fibrosis.

This was a randomised, double-blind, placebo-controlled study. Primary outcome measures were sputum neutrophil count, lung function, 24-h sputum weight, BronkoTest ${ }_{\mathbb{R}}$ diary card data and health-related quality-of-life (revised cystic fibrosis quality-of-life questionnaire). Secondary end-points included sputum neutrophil elastase activity, inflammatory biomarkers in sputum and blood, urine and plasma desmosine (an elastin degradation marker), AZD9668 levels and safety parameters (adverse events, routine haematology, biochemistry, electrocardiogram and sputum bacteriology).

56 patients were randomised, of which 27 received AZD9668. There was no effect for AZD9668 on sputum neutrophil counts, neutrophil elastase activity, lung function or clinical outcomes, including quality of life. In the AZD9668 group, there was a trend towards reduction in sputum inflammatory biomarkers with statistically significant changes in interleukin-6, RANTES and urinary desmosine. The pattern of adverse events was similar between groups.

Consistent reductions in sputum inflammatory biomarkers were seen in the AZD9668 group, and reduction in urinary desmosine suggests that AZD9668 impacts elastin cleavage by neutrophil elastase.

KEYWORDS: Inflammatory lung disease, neutrophil elastase, tissue damage

B ronchiectasis, chronic obstructive pulmonary disease (COPD) and cystic fibrosis (CF) are inflammation- and injury-driven respiratory conditions with significant unmet needs.

CF results from airway obstruction by dehydrated and thickened secretions and chronic endobronchial infections [1,2]. Chronic infection is associated with an exaggerated inflammatory response, with high levels of the pro-inflammatory cytokine interleukin (IL)-8 in the airways and neutrophil infiltration of the lungs [3,4]. The presence of neutrophils in the airways, and the resulting high concentrations of neutrophil proteases, suggest that they are contributors in the pathogenesis of proteolytic lung destruction associated with CF $[1,3,4]$.

Neutrophil elastase (NE) is a key proteolytic enzyme implicated in the pathogenesis and progression of neutrophil-driven inflammatory lung diseases. It is pro-inflammatory $[5,6]$, stimulates mucus overproduction [7-9], impairs mucociliary clearance perpetuating the neutrophilic inflammation
$[1,3]$ and directly causes lung tissue damage $[10,11]$. The use of NE inhibitors, such as $\alpha_{1}$ antitrypsin $\left(\alpha_{1}-\mathrm{AT}\right)$ in patients with CF [4, 12], is therefore a logical approach to treating these conditions and offers the potential to address the current unmet medical needs.

AZD9668 is an oral inhibitor of human NE. Preclinical studies have shown that it reversibly inhibits NE activity, is highly potent and selective in biochemical and cellular assays and shows efficacy in in vivo models of lung inflammation and degradation [13]. The favourable pharmacokinetic profile, allowing oral administration, and the decreased risk of toxicity due to its reversible interaction with the target enzyme suggest that AZD9668 represents a significant advance versus previous NE inhibitors [13]. As such, AZD9668 was developed as a possible therapeutic agent for symptomatic treatment and disease modification in COPD, but may also be of benefit in $\mathrm{CF}$ and other airway diseases characterised by neutrophilic inflammation.

\section{AFFILIATIONS}

${ }^{*}$ Respiratory Medicine Group, Centre for Infection and Immunity, Queen's University, Belfast, and

${ }^{\#}$ Soluble Biomarkers - Science and Validation, AstraZeneca, Loughborough, UK. "Bioscience Dept, AstraZeneca, Lund, Sweden.

CORRESPONDENCE

J.S. Elborn

Respiratory Medicine Group, Health Sciences Building, Centre for Infection and Immunity Queen's University of Belfast 97 Lisburn Road Belfast BT9 7BC UK E-mail: s.elborn@qub.ac.uk

Received:

Nov 082011

Accepted after revision: Jan 032012

First published online:

Jan 202012 
As CF is an orphan disease, efficient and appropriate assessment of new therapies in the limited number of available patients is important. Frequently used outcome measures include clinical efficacy measures (survival, pulmonary exacerbations and hospitalisations), surrogate end-points (forced expiratory volume in $1 \mathrm{~s}$ (FEV1) at a given time point, or the rate of decline in FEV1) and biomarkers [2]. Given that proteolytic lung destruction is a key feature of $\mathrm{CF}$, there is also a need for relevant, reproducible markers of injury/tissue degradation to enable assessment of the effectiveness of novel treatments.

The aim of this signal-searching study was to make a preliminary evaluation of the efficacy and safety of AZD9668 versus placebo in patients with $\mathrm{CF}$, and to examine its effect on biomarkers of inflammation and tissue damage.

\section{METHODS}

\section{Study design}

This was a randomised, double-blind, placebo-controlled, parallelgroup, phase IIa study (NCT00757848). After a run-in period of up to 4 weeks, patients were randomised to 28 days of treatment with AZD9668 or matching placebo.

\section{Patients}

Male and female (post-menopausal or surgically sterile) patients $\geqslant 16$ yrs, with a clinical diagnosis of CF, a FEV $1 \geqslant 40 \%$ predicted value, normal laboratory values and normal renal function (glomerular filtration rate of $>70 \mathrm{~mL} \cdot \mathrm{min}^{-1}$ ) were eligible.

Exclusion criteria included any non-CF-related lung disease which could interfere with study assessments.

\section{Treatments}

Patients were randomised (1:1) via a computer-generated randomisation scheme, to AZD9668 $60 \mathrm{mg}$ b.i.d. or matching placebo. The dose selected was close to the maximum used in prior healthy volunteer studies with AZD9668 [14]. Standard CF therapies were continued; oral corticosteroids and nonprophylactic antibiotics were not allowed during the study and for 8 and 4 weeks, respectively, prior to randomisation.

\section{Assessments}

Primary end-points were: sputum absolute and percentage neutrophil counts; $24 \mathrm{~h}$ sputum weight; lung function; BronkoTest diary card data; and quality of life (QoL) measured by the revised CF QoL questionnaire (CFQ-R) [15].

Secondary end-points were: sputum NE activity; sputum inflammatory biomarkers; blood inflammatory biomarkers; urinary desmosine; safety; and AZD9668 concentration in plasma and induced sputum. Plasma desmosine was an exploratory outcome.

For sputum biomarkers, the mean of the two induced sputum samples obtained at baseline (visits 1a and 2) was compared with the mean of the two samples taken during the last week of treatment (visits $3 a$ and 4) (fig. 1). The mean value of the two measurements was used to reduce the variance of the estimates of the biomarkers.

\section{Statistical analyses}

For neutrophil count and biomarker data ANCOVA was used to determine the ratio of AZD9668:placebo. Baseline data (log scale) and country were included as covariates. For other efficacy

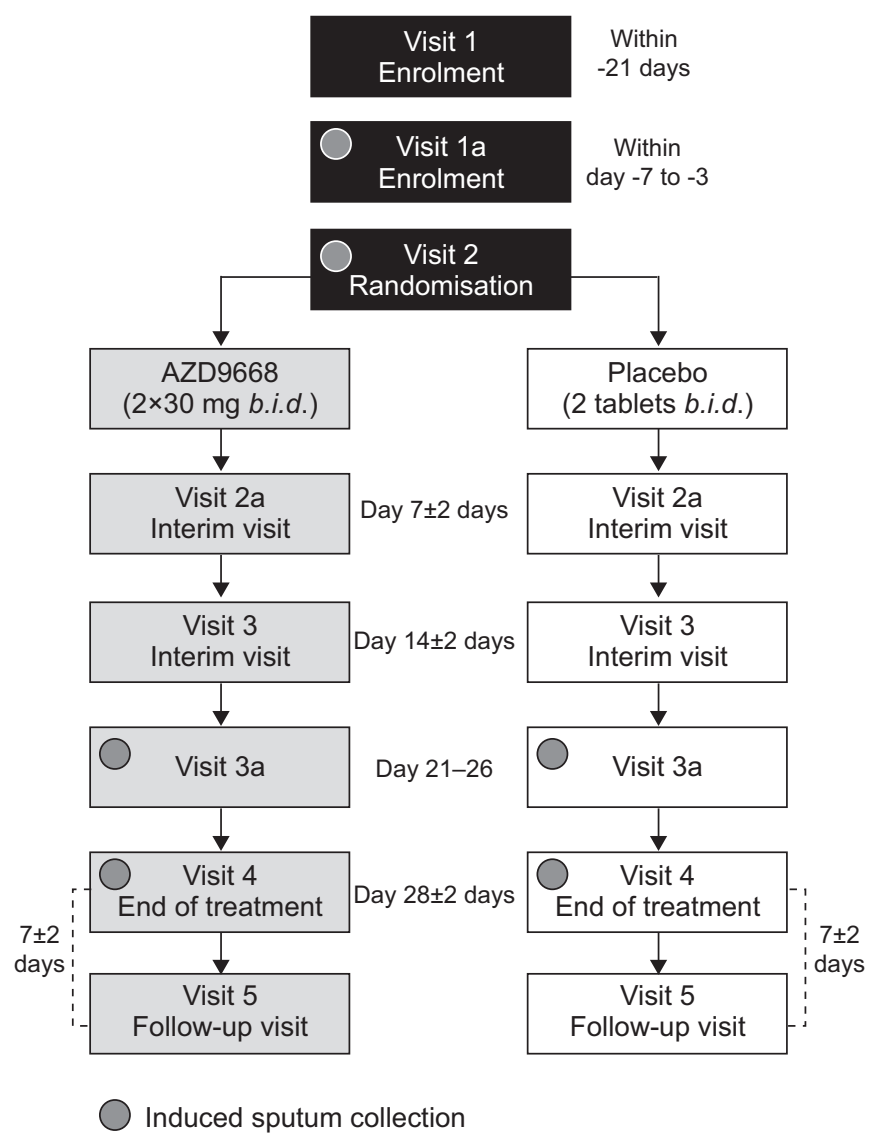

FIGURE 1. Flow chart of the study design.

outcomes, ANCOVA was used to analyse differences in the change from baseline to day 28 between AZD9668 and placebo, where treatment and country were fixed factors and baseline values were the covariate.

As this was an exploratory study, sample size was not based on obtaining power to detect specific effects. Assuming a standard deviation of 1 for logged data, such that a $50 \%$ decrease in neutrophil numbers in the sputum would not be missed $(80 \%$ power), a sample size of 40 patients (20 per treatment group) was considered sufficient and a two-sided $p$-value $<0.1$ statistically significant. There was no adjustment for multiplicity of tests.

\section{Ethical aspects}

The study protocol and amendments were approved by an independent ethics committee and were in accordance with the Declaration of Helsinki and the International Conference on Harmonisation/good clinical practice. All patients provided their informed consent.

Further methodological detail on exclusion criteria, prohibited treatments, sputum sampling, biomarker analysis and assessment timelines can be found in the online supplementary material.

\section{RESULTS}

\section{Patients}

The first patient was enrolled on October 30, 2008, and the last patient completed the study on August 4, 2009. A total of 70 


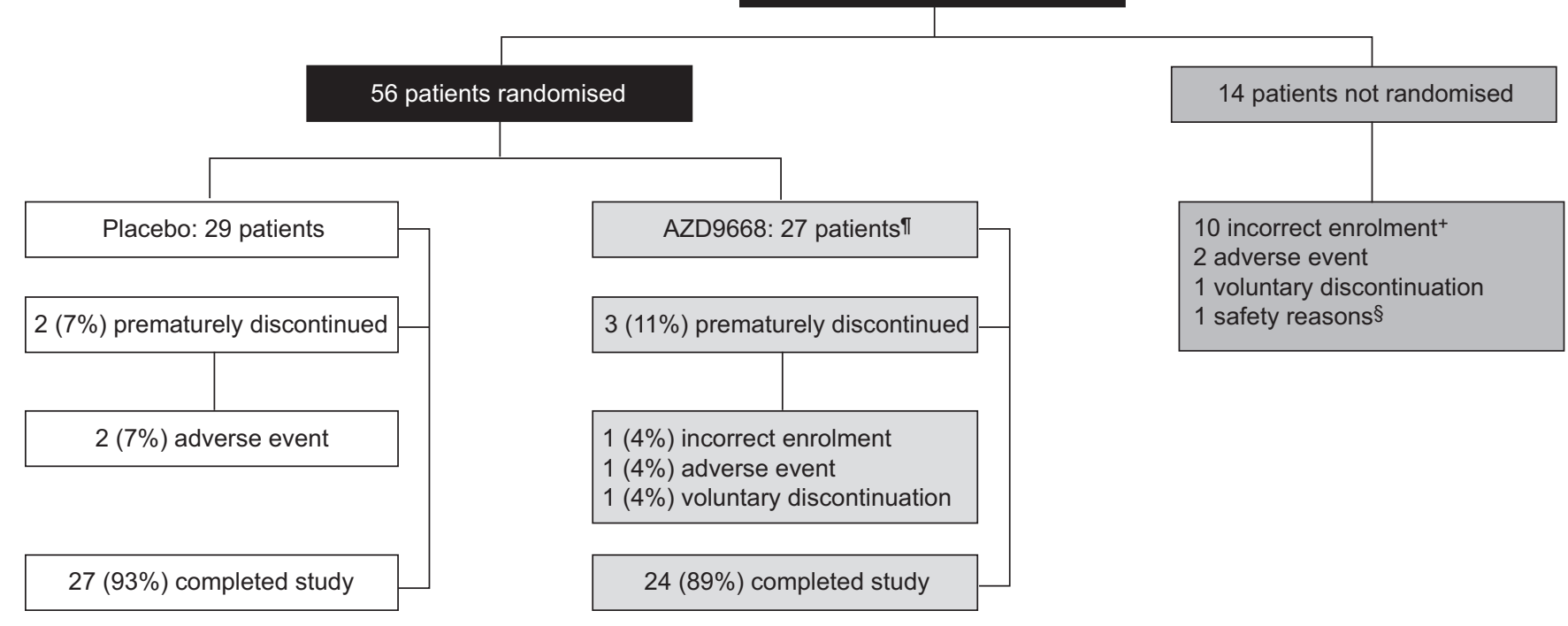

FIGURE 2. The disposition of patients. "\#: includes a patient who was enrolled twice; " : one patient did not take a dose (safety analysis set, $n=26)$, another patient had no post-dose efficacy data (efficacy analysis set, $\mathrm{n}=25$ ); ${ }^{+}$: failure to meet the inclusion/exclusion criteria; ${ }^{\S}$ : patient had elevated alanine aminotransferase.

patients were enrolled in the study and 56 were randomised to treatment; 29 received placebo and 27 received AZD9668 $60 \mathrm{mg}$ b.i.d. Of those patients randomised to treatment, $27(93 \%)$ placebo patients and 24 (89\%) AZD9668 patients completed the study. The disposition of patients is shown in figure 2 .

All patients in the placebo group received at least one dose of study treatment and had post-dose efficacy data (safety and efficacy analysis sets, $n=29$ ). One patient in the AZD9668 group did not receive any study medication following randomisation and was excluded from the safety and efficacy analysis sets. A further patient in the AZD9668 group did not have post-dose efficacy data and was excluded from the efficacy analysis set. Therefore, for the AZD9668 group, the safety analysis set had 26 patients and the efficacy analysis set had 25.

Overall, the two treatment groups were well matched for the majority of the demographic and patient characteristics (table 1). There was only one female in the study. FEV1 was lower, both at screening and randomisation, in the group receiving AZD9668.

\section{Primary outcome variables}

Absolute and percent neutrophil counts

At the end of treatment, the geometric mean absolute neutrophil count was lower in the placebo group $\left(11.13 \times 10^{6} \cdot \mathrm{g}^{-1}\right)$ than in the AZD9668 group $\left(14.10 \times 10^{6} \cdot \mathrm{g}^{-1}\right)$; however, the ratio between groups was not statistically significantly different: 0.97 (90\% CI 0.69-1.38; $\mathrm{p}=0.891$ ) (table 2). Similarly, there was no statistically significant difference between AZD9668 and placebo for percentage neutrophil count at the end of treatment; least squares mean difference $1.25 \%(90 \%$ CI $-2.53-5.04 ; p=0.581)$ (table 3). Individual patient data for the changes from baseline in neutrophil counts are shown in online supplementary figure E1.

\section{Sputum weight and lung function}

Analysis of 24-h sputum weight, FEV1, slow vital capacity, forced vital capacity (FVC), forced expiratory flow at $25-75 \%$ of FVC and FEV1 \% pred showed no statistically significant differences between groups (online supplementary table E1 and fig. E2) and there was no difference in lung function variables measured daily at home (BronkoTest diary card data) (online supplementary table E1).

\begin{tabular}{|c|c|c|c|}
\hline \multirow{3}{*}{\multicolumn{2}{|c|}{$\begin{array}{l}\text { Demographic and ke } \\
\text { efficacy analysis set }\end{array}$}} & baseline & racteristics: \\
\hline & & \multicolumn{2}{|c|}{ Treatment group } \\
\hline & & Placebo & $\begin{array}{l}\text { AZD9668 } \\
60 \mathrm{mg} \text { b.i.d. }\end{array}$ \\
\hline Subjects $n$ & & 29 & 25 \\
\hline Age yrs & & $27 \pm 8.5$ & $29 \pm 10.0$ \\
\hline \multicolumn{4}{|l|}{ Sex } \\
\hline Male & & $29(100)$ & $24(96)$ \\
\hline Female & & $0(0)$ & $1(4)$ \\
\hline \multicolumn{4}{|l|}{ Race } \\
\hline White & & $29(100)$ & $25(100)$ \\
\hline$B M I \mathbf{k g} \cdot \mathrm{m}^{-2}$ & edian (range) & $22.2(3.2)$ & $22.4(3.0)$ \\
\hline \multicolumn{4}{|c|}{ Nicotine use $e^{\#}$} \\
\hline Never & & $24(83)$ & $24(92)$ \\
\hline Current & & $4(14)$ & $1(4)$ \\
\hline Former & & $1(3)$ & $1(4)$ \\
\hline Number of & ack-yrs & $6 \pm 5.4$ & $4 \pm 5.7$ \\
\hline FEV $1 \%$ pred & & $78.4 \pm 23.7$ & $65.7 \pm 20.0$ \\
\hline Cystic fibros & duration yrs ${ }^{\#}$ & $19.8 \pm 10.4$ & $23.4 \pm 10.8$ \\
\hline Pancreatic ir & sufficiency ${ }^{\#}$ & $26(90)$ & $23(88)$ \\
\hline Cystic fibros & related diabetes mellitus ${ }^{\#}$ & $4(14)$ & $5(19)$ \\
\hline
\end{tabular}




\begin{tabular}{|c|c|c|c|c|c|c|}
\hline & Placebo & AZD9668 & Placebo & AZD9668 & Ratio $(90 \% \mathrm{Cl})$ & p-value \\
\hline Subjects $n$ & 28 & 24 & 28 & 24 & & \\
\hline
\end{tabular}

Signs and symptoms of CF

There were no statistically significant differences between the treatment groups for respiratory symptom scores or use of reliever medication (online supplementary table E1). During the study, antibiotics/steroids were used by up to seven $(24 \%)$ patients in the placebo group and $10(40 \%)$ in the AZD9668 group. Concomitant medication use is summarised in the online supplementary material. There were no statistically significant changes in individual CFQ-R categories with the exception of "emotion" $(p<0.05)$ and "eat" $(p<0.1)$, where deteriorations were recorded in the AZD9668 group (online supplementary table E1).

\section{Secondary outcome variables}

NE activity in sputum

Geometric mean baseline NE activity was higher in the AZD9668 than the placebo group. The ratios of NE activity at the end of treatment showed an increase (51\%) in the placebo group and a decrease (5\%) in the AZD9668 group. There was a 37\% reduction in NE activity in the AZD9668 group versus the placebo group (ratio $0.63 ; 90 \%$ CI $0.30-1.31$ ); however, this was not statistically significant $(p=0.292)$ (table 4). Individual patient data for the changes from baseline in NE activity are shown in online supplementary figure E3.

\section{Inflammatory biomarkers}

Overall, there was a trend towards a reduction in all of the measured sputum inflammatory biomarkers except leukotriene $\mathrm{B}_{4}\left(\mathrm{LTB}_{4}\right)$ (i.e. tumour necrosis factor (TNF)- $\alpha$, IL-6, IL-8, IL- $1 \beta$, RANTES (regulated upon activation, normal T-cell expressed and secreted) and monocyte chemoattractant protein-1) in the AZD9668 group and the changes were statistically significant for IL-6 ( $\mathrm{p}<0.01)$ and RANTES ( $\leqslant 0.1)$ (fig. 3; online supplementary table E2). There were no statistically significant differences between the groups in changes in blood inflammatory biomarkers (data not shown).

\section{Desmosine}

Desmosine at baseline and changes from baseline are shown in figure $4 \mathrm{a}$ and $\mathrm{b}$. Statistically significant reductions in both free and total urinary desmosine occurred in the AZD9668 group; free and total urine desmosine (normalised for creatinine) were reduced by $30 \%(p<0.01)$ and $31 \%(p<0.05)$, respectively (table 4 and fig. 4c). Plasma desmosine was also reduced by $16 \%$ in the AZD9668 group compared with placebo but this reduction was not statistically significant $(\mathrm{p}=0.146)$.

\section{Safety}

Overall, 14 (48\%) patients reported treatment-emergent adverse events (AEs) in the placebo group, compared with $12(46 \%)$ in the AZD9668 group (online supplementary table E3); in total 54 and 32 AEs were reported in the placebo and AZD9668 groups, respectively.

Two patients, both in the placebo group, reported serious AEs (pulmonary exacerbation and pneumonia) and two patients in the placebo group discontinued treatment due to an $\mathrm{AE}$ (noncardiac chest pain and pneumonia). There were no deaths or other significant AEs (online supplementary table E3).

In general, the $\mathrm{AE}$ profile (by preferred term) was similar between the treatment groups. The most commonly reported $\mathrm{AE}$ was headache, which was reported by a greater number of patients in the AZD9668 group than in the placebo group (online supplementary table E3).

There were no clinically significant differences between groups in sputum bacteriology, haematology or clinical chemistry parameters, vital signs, electrocardiogram or physical examinations.

TABLE 3 Effect of AZD9668 $60 \mathrm{mg}$ b.i.d. versus placebo on differential neutrophil counts up to day 28: efficacy analysis set

\begin{tabular}{|c|c|c|c|c|c|c|}
\hline & \multicolumn{2}{|c|}{ Baseline } & \multicolumn{2}{|c|}{ End of treatment } & \multicolumn{2}{|c|}{ Difference between AZD9668 and placebo } \\
\hline & Placebo & AZD9668 & Placebo & AZD9668 & LSM $\pm \operatorname{SEM}(90 \% \mathrm{Cl})$ & $\mathrm{p}$-value \\
\hline Subjects $n$ & 28 & 24 & 28 & 24 & & \\
\hline Sputum neutrophils \% & $86.28 \pm 22.28$ & $95.41 \pm 5.67$ & $90.19 \pm 16.63$ & $96.48 \pm 3.93$ & $1.25 \pm 2.25(-2.53-5.04)$ & 0.581 \\
\hline
\end{tabular}


TABLE 4 Effect of AZD9668 $60 \mathrm{mg}$ b.i.d. versus placebo on neutrophil elastase activity and desmosine levels at end of treatment up to day 28: safety analysis set

\begin{tabular}{|c|c|c|c|c|c|c|}
\hline & \multicolumn{2}{|c|}{ Baseline } & \multicolumn{2}{|c|}{ End of treatment } & \multicolumn{2}{|c|}{$\begin{array}{c}\text { Ratio of AZD9668 to placebo } \\
\text { at end of treatment }\end{array}$} \\
\hline & Placebo & AZD9668 & Placebo & AZD9668 & Ratio $(90 \% \mathrm{Cl})$ & p-value \\
\hline Subjects & 29 & 26 & 29 & 26 & & \\
\hline Neutrophil elastase activity $\mu \mathrm{mol} \cdot \mathrm{L}^{-1} \mathrm{AMC} \cdot \mathrm{h}^{-1}$ & $55.72(4275)$ & $154.7(400)$ & $106.7(746)$ & $148.4(375)$ & $0.63(0.30-1.31)$ & 0.292 \\
\hline Subjects & 25 & 21 & 25 & 21 & & \\
\hline Total desmosine (urine) ${ }^{\#} \mathrm{nmol} \cdot \mathrm{mmol}^{-1}$ & $2.56(64.41)$ & $2.56(53.01)$ & $2.67(104.3)$ & $1.87(44.62)$ & $0.69(0.51-0.93)$ & 0.044 \\
\hline Subjects & 27 & 22 & 27 & 22 & & \\
\hline Desmosine (plasma) pmol $\cdot \mathrm{L}^{-1}$ & $85.70(60.47)$ & $75.08(59.62)$ & $92.86(70.90)$ & $69.61(49.86)$ & $0.84(0.69-1.02)$ & 0.146 \\
\hline Subjects & 27 & 23 & 27 & 23 & & \\
\hline
\end{tabular}

Data are presented as $\mathrm{n}$, geometric mean coefficient of variation (\%), unless otherwise stated. AMC: 7-amino-4-methylcoumarin. \#: desmosine adjusted for creatinine.

One patient in the AZD9668 group showed an increase in creatinine phosphokinase, alanine transaminase, aspartate transaminase and lactic dehydrogenase, peaking on days 7 and 28 of treatment. Heavy exercise was temporally associated with the initial increase; however, insufficient information was available to explain the second increase at day 28 , meaning that a relationship to the study drug cannot be excluded.

\section{AZD9668 in sputum and plasma}

Concentrations of AZD9668 measured in plasma and sputum supernatant samples confirmed that the AZD9668 levels were comparable to a previous study in patients with bronchiectasis [16].

\section{DISCUSSION}

Treatment with AZD9668 $60 \mathrm{mg}$ b.i.d. over 28 days had no effect on symptoms, lung function, sputum neutrophil count, sputum weight or sputum NE activity in patients with $\mathrm{CF}$

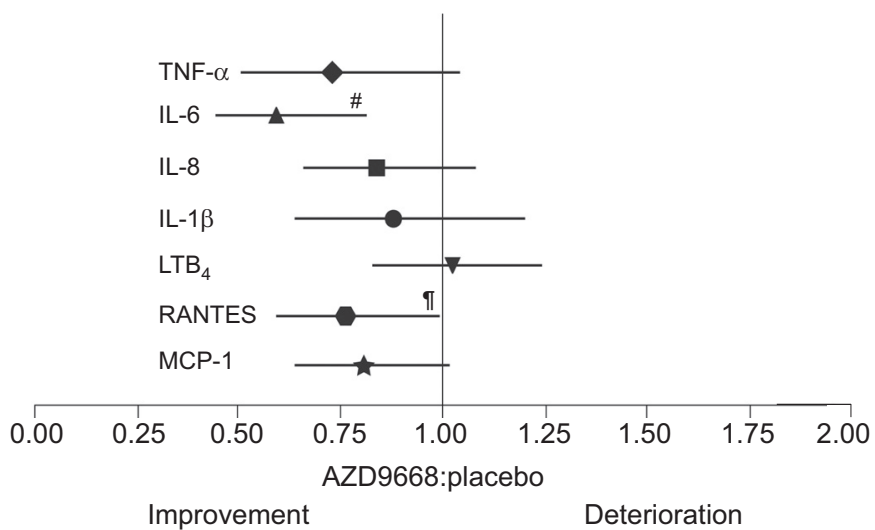

FIGURE 3. Ratio $(90 \% \mathrm{Cl})$ for AZD9668 to placebo for the inflammatory biomarkers in induced sputum. TNF: tumour necrosis factor; IL: interleukin; LTB leukotriene B; RANTES: regulated upon activation, normal T-cell expressed and secreted; MCP: monocyte chemoattractant protein. ${ }^{\natural:} p=0.006 ;{ }^{*}: p=0.1$ versus placebo. compared with placebo. However, with the exception of $\mathrm{LTB}_{4}$ AZD9668 treatment did result in a consistent pattern of reduction in sputum inflammatory biomarkers, which was statistically significant for IL-6 and RANTES. AZD9668 treatment also significantly decreased free and total urine desmosine, biomarkers of elastin degradation. There was also a reduction in plasma desmosine in the AZD9668 group, although the

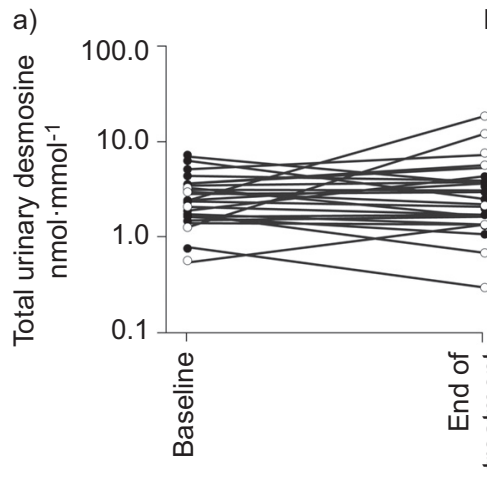

Treatment period b)

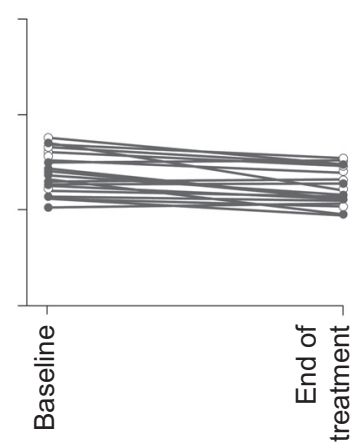

Treatment period c)

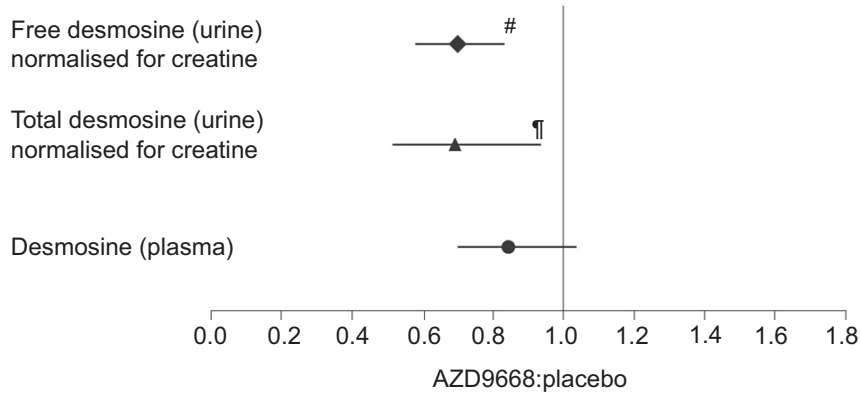

Improvement

Deterioration

FIGURE 4. Individual patient data for urinary desmosine in patients randomised to a) placebo and b) AZD9668. c) Ratio $(90 \% \mathrm{Cl})$ for AZD9668 to placebo for urinary and plasma desmosine. ${ }^{\circ}: p=0.44 ;{ }^{\#}: p=0.002$ versus placebo. 
difference versus placebo was not significant. AZD9668 was generally well tolerated throughout.

Given the proteolytic activity of sputum in CF disease, there is significant interest in identifying biomarkers that indicate the extent of airway inflammation and lung tissue damage in CF. A biomarker of structural injury may enable earlier intervention and the ability to monitor lung tissue changes during the course of the disease. Desmosine, a cross-linking amino acid present in elastin is released during matrix degradation and can be detected in sputum, blood and urine of COPD patients [17-20] and has been considered as a potential biomarker of lung tissue injury. Our study demonstrated consistent reductions in urinary and plasma desmosine in response to AZD9668 treatment and is one of few studies where a therapeutic agent has shown a positive effect on desmosine levels, suggesting that AZD9668 may have disease-modifying potential in neutrophil-driven disease states. A related NE inhibitor, ZD0892, also reduced desmosine levels in lavage fluid in a guinea pig model of smokeinduced emphysema [11]. Another CF patient study measured desmosine in the sputum of patients hospitalised for treatment of a pulmonary exacerbation and found that sputum desmosine levels decreased significantly during the first week of hospitalisation $(p=0.04)$ [21]. Desmosine levels were also positively correlated with decreases in plasma $C$-reactive protein $(p=0.03)$, sputum IL-8 $(\mathrm{p}<0.01)$ and sputum NE protein $(\mathrm{p}<0.01)$. In a study of 39 patients with CF, DOWNEY et al. [22] showed an inverse correlation between urinary desmosine levels and survival, where analysis of data taken from patients $1 \mathrm{yr}$ after the start of the study showed significantly higher levels of urinary desmosine in non-survivors compared with survivors $(\mathrm{p}<0.0001)$. There is also evidence that the urinary excretion of desmosine is increased in smokers and in COPD patients [20, 23, 24]. A recent study from the Swedish Twin Registry evaluated the relationship between desmosine and lung function [25]. This study found that urinary desmosine was significantly and inversely correlated with FEV1, FVC and the diffusion capacity for carbon monoxide, and positively correlated with residual volume (RV) and RV/total lung capacity, and that the correlations were markedly stronger in subjects with COPD.

The current study also showed that AZD9668 treatment resulted in a consistent pattern of reduction in sputum inflammatory biomarkers. No treatment effect was observed for plasma inflammatory biomarkers. The results for the plasma inflammatory biomarkers are consistent with the assessments in the Evaluation of COPD Longitudinally to Identify Predictive Surrogate Endpoints (ECLIPSE) study, which reported weak associations between neutrophil measurements in sputum and blood and systemic biomarkers [26]. Biomarker analysis in studies of NE inhibitors is also challenging because appropriate biomarkers for inhibition of NE associated with a clinically meaningful anti-inflammatory effect are currently unknown.

The lack of effect of AZD9668 on neutrophil counts, NE activity and lung function measurements observed in the current study could be for various reasons. Our study was a short, signalsearching, proof-of-principle study, carried out in a small number of patients, and was associated with large inter-individual variation resulting in large confidence intervals. The study duration was most likely too short and not powered to investigate diseasemodifying effects of AZD9668. Longer study durations are often needed before an impact on lung function and end-points such as QoL can be detected.

Previous analyses in patients with bronchiectasis have demonstrated the presence of AZD9668 in plasma and sputum supernatants at expected concentrations [16], and the measured concentrations of AZD9668 in plasma and sputum in our study confirmed exposure at pharmacologically relevant levels, predicted to inhibit NE. However, one possibility is that higher concentrations of AZD9668 would be required to reach a sufficient level of NE inactivation in the airways. Other possibilities are that AZD9668 may not be reaching its site of action or being inactivated at its site of action. It is known that inactivation of endogenous inhibitors of NE, such as $\alpha_{1}-\mathrm{AT}$, can occur due to oxidants present in lung tissue $[27,28]$, although it is unclear whether a small molecule drug is susceptible to similar inactivation processes in patients.

Another possible reason for the lack of an effect could be related to target specificity. AZD9668 has been shown to selectively target NE [13]; however, airway inflammation and proteolytic activity are driven by a number of different mechanisms and several other neutrophilic proteases. For example, high levels of cathepsin $\mathrm{G}$ and proteinase 3 have been identified in bronchoalveolar lavage fluid of CF patients $[29,30]$. In addition, the neutrophil matrix metalloproteases collagenase (MMP-8) and gelatinase (MMP-9), released under inflammatory conditions, have also been detected in high concentrations in the bronchoalveolar lavage fluid of CF patients [31, 32]. As AZD9668 demonstrates in vivo anti-inflammatory effects [13], it is possible that it could affect NE-independent anti-inflammatory pathways leading to a reduction in inflammatory biomarkers, as well as a reduction in the levels of desmosine.

A further explanation is that NE damages the extracellular matrix in the process of transmigration. It is generally assumed that the high concentrations of NE result in proteolytic damage to matrix proteins. However, as elastase is released during neutrophil migration from the circulation into the airway, it is possible that due to increased priming the neutrophils release more NE or that the increased number of neutrophils results in more NE. It is therefore possible that AZD9668 is more active in the extracellular matrix than in the airway where concentrations of NE are much greater. This could account for the reduction in desmosine concentrations in the absence of much change in neutrophil numbers or NE activity in the airway. Alternatively, the complex biology of the sputum matrix could have played a role in the high inter-patient variability of the sputum NE assay. This variability, together with a small sample size, could, in part, explain the trend towards reduced NE activity but lack of statistical significance.

Our results suggest that AZD9668 is safe and well tolerated in patients with CF and has a consistent impact on sputum biomarkers of inflammation, though little effect on NE activity or lung function. These data are similar to those from other studies investigating inhibitors of NE. One pilot study, investigating the safety and efficacy of inhaled recombinant human $\alpha_{1}$-AT (an endogenous inhibitor of NE) as a treatment for $\mathrm{CF}$, found that nebulised recombinant $\alpha_{1}$-AT was safe and well tolerated, but had a limited effect on absolute sputum NE levels, NE activity, and other markers of inflammation [12]. Another phase II trial in 
CF suggested no effect of $\alpha_{1}$-AT on pulmonary function (FEV1) but did result in an increase in $\alpha_{1}$-AT levels and decreases in free NE activity, neutrophil numbers, Pseudomonas aeruginosa colony forming units, IL- 8 and TNF- $\alpha$ levels and intact immunoglobulin G [4].

The results of the current study are suggestive of an effect of AZD9668 in inflammatory respiratory diseases with a neutrophildriven component such as CF. Studies of longer duration including more patients are needed to further investigate the impact of AZD9668 on neutrophil numbers, NE activity and lung function, and to confirm the effectiveness of NE inhibition as a therapeutic approach. In addition, these results point to the potential usefulness of desmosine as a biomarker of lung tissue degradation.

\section{SUPPORT STATEMENT}

This study was funded by AstraZeneca.

\section{CLINICAL TRIAL}

This study is registered at www.ClinicalTrials.gov with identifier number NCT00757848

\section{STATEMENT OF INTEREST}

Statements of interest for all authors of this manuscript and for the study itself can be found at www.erj.ersjournals.com/site/misc/ statements.xhtml

\section{ACKNOWLEDGEMENTS}

We thank the investigators and patients for their participation in this study. Principal investigators: T. Pressler (Rigshospitalet, Copenhagen, Denmark); M. Griese (Ludwig Maximilian University, Munich, Germany); R. Fischer (Munich University Hospital, Munich, Germany); H-E. Heuer (Othmarschen Park Children And Youth Medical Practice, Hamburg, Germany); H. Wirtz (Leipzig University Hospital, Leipzig, Germany); B. Bewig (Schleswig-Holstein University Hospital, Kiel, Germany); H. Mazurek (Institute of Tuberculosis and Lung Diseases, Warsaw, Poland); E. Amelina (Roszdrav Pulmonology Research Institute, Russia); F. Karpati (Karolinska University Hospital, Stockholm, Sweden); L. Mared (Lund University Hospital, Lund, Sweden); A. Hollsing (Uppsala University Hospital, Uppsala, Sweden); A. Lindblad (Gothenburg Children's Hospital, Gothenburg, Sweden); M. Gilljam (Sahlgrenska University Hospital, Gothenburg, Sweden); S. Elborn (Belfast City Hospital, Belfast, UK); M. Walshaw (Liverpool Heart and Chest Hospital, Liverpool, UK).

Medical writing assistance was provided by V. Munoz from Complete Medical Communications, Macclesfield, UK.

\section{REFERENCES}

1 Mayer-Hamblett N, Aitken ML, Accurso FJ, et al. Association between pulmonary function and sputum biomarkers in cystic fibrosis. Am J Respir Crit Care Med 2007; 175: 822-828.

2 Mayer-Hamblett N, Ramsey BW, Kronmal RA. Advancing outcome measures for the new era of drug development in cystic fibrosis. Proc Am Thorac Soc 2007; 4: 370-377.

3 Voynow JA, Fischer BM, Zheng S. Proteases and cystic fibrosis. Int J Biochem Cell Biol 2008; 40: 1238-1245.

4 Griese M, Latzin P, Kappler M, et al. $\alpha_{1}$-Antitrypsin inhalation reduces airway inflammation in cystic fibrosis patients. Eur Respir J 2007; 29: 240-250.

5 Bergin DA, Greene CM, Sterchi EE, et al. Activation of the epidermal growth factor receptor (EGFR) by a novel metalloprotease pathway. J Biol Chem 2008; 283: 31736-31744.
6 Chen HC, Lin HC, Liu CY, et al. Neutrophil elastase induces IL-8 synthesis by lung epithelial cells via the mitogen-activated protein kinase pathway. J Biomed Sci 2004; 11: 49-58.

7 Amitani R, Wilson R, Rutman A, et al. Effects of human neutrophil elastase and Pseudomonas aeruginosa proteinases on human respiratory epithelium. Am J Respir Cell Mol Biol 1991; 4: 26-32.

8 Caldwell RA, Boucher RC, Stutts MJ. Neutrophil elastase activates near-silent epithelial $\mathrm{Na}+$ channels and increases airway epithelial Na+ transport. Am J Physiol Lung Cell Mol Physiol 2005; 288: L813-L819.

9 Voynow JA, Fischer BM, Malarkey DE, et al. Neutrophil elastase induces mucus cell metaplasia in mouse lung. Am J Physiol Lung Cell Mol Physiol 2004; 287: L1293-L1302.

10 Kuraki $\mathrm{T}$, Ishibashi M, Takayama M, et al. A novel oral neutrophil elastase inhibitor (ONO-6818) inhibits human neutrophil elastaseinduced emphysema in rats. Am J Respir Crit Care Med 2002; 166: 496-500.

11 Wright JL, Farmer SG, Churg A. Synthetic serine elastase inhibitor reduces cigarette smoke-induced emphysema in guinea pigs. Am J Respir Crit Care Med 2002; 166: 954-960.

12 Martin SL, Downey D, Bilton D, et al. Safety and efficacy of recombinant $\alpha(1)$-antitrypsin therapy in cystic fibrosis. Pediatr Pulmonol 2006; 41: 177-183.

13 Stevens T, Ekholm K, Granse M, et al. AZD9668: Pharmacological characterisation of a novel oral inhibitor of neutrophil elastase. J Pharmacol Exp Ther 2011; 339: 313-320.

14 Gunawardena K, Gullstrand H, Perrett J. Safety, tolerability and pharmacokinetics of AZD9668, an oral neutrophil elastase inhibitor, in healthy subjects and patients with COPD. Eur Respir J 2010; 38: Suppl. 54, 203s.

15 Quittner AL, Sweeny S, Watrous M, et al. Translation and linguistic validation of a disease-specific quality of life measure for cystic fibrosis. J Pediatr Psychol 2000; 25: 403-414.

16 Stockley R, Snell N, Perrett J, et al. Efficacy and safety of AZD9668, an oral neutrophil elastase inhibitor, in idiopathic bronchiectasis. Eur Respir J 2010; 38: Suppl. 54, 38s.

17 Ma S, Lieberman S, Turino GM, et al. The detection and quantitation of free desmosine and isodesmosine in human urine and their peptide-bound forms in sputum. Proc Natl Acad Sci USA 2003; 100: 12941-12943.

18 Ma S, Lin YY, Turino GM. Measurements of desmosine and isodesmosine by mass spectrometry in COPD. Chest 2007; 131: 1363-1371.

19 Ma S, Lin YY, Tartell L, et al. The effect of tiotropium therapy on markers of elastin degradation in COPD. Respir Res 2009; 10: 12.

20 Stone PJ, Gottlieb DJ, O'Connor GT, et al. Elastin and collagen degradation products in urine of smokers with and without chronic obstructive pulmonary disease. Am J Respir Crit Care Med 1995; 151: 952-959.

21 Laguna TA, Wagner BD, Luckey HK, et al. Sputum desmosine during hospital admission for pulmonary exacerbation in cystic fibrosis. Chest 2009; 136: 1561-1568.

22 Downey DG, Martin SL, Dempster M, et al. The relationship of clinical and inflammatory markers to outcome in stable patients with cystic fibrosis. Pediatr Pulmonol 2007; 42: 216-220.

23 Viglio S, Iadarola P, Lupi A, et al. MEKC of desmosine and isodesmosine in urine of chronic destructive lung disease patients. Eur Respir J 2000; 15: 1039-1045.

24 Harel S, Janoff A, Yu SY, et al. Desmosine radioimmunoassay for measuring elastin degradation in vivo. Am Rev Respir Dis 1980; 122: 769-773.

25 Lindberg C, Engström G, Gerhardsson de Verdier M, et al. Total desmosines in plasma and urine correlate with lung function. Eur Respir J 2011; 39: 839-845.

26 Singh D, Edwards L, Tal-Singer R, et al. Sputum neutrophils as a biomarker in COPD: findings from the ECLIPSE study. Respir Res 2010; $11: 77$. 
27 Johnson D, Travis J. The oxidative inactivation of human $\alpha-1$ proteinase inhibitor. Further evidence for methionine at the reactive center. J Biol Chem 1979; 254: 4022-4026.

28 Zeiher BG, Matsuoka S, Kawabata K, et al. Neutrophil elastase and acute lung injury: prospects for sivelestat and other neutrophil elastase inhibitors as therapeutics. Crit Care Med 2002; 30: Suppl. 5, S281-S287.

29 Goldstein W, Doring G. Lysosomal enzymes from polymorphonuclear leukocytes and proteinase inhibitors in patients with cystic fibrosis. Am Rev Respir Dis 1986; 134: 49-56.
30 Witko-Sarsat V, Halbwachs-Mecarelli L, Schuster A, et al. Proteinase 3, a potent secretagogue in airways, is present in cystic fibrosis sputum. Am J Respir Cell Mol Biol 1999; 20: 729-736.

31 Ratjen F, Hartog CM, Paul K, et al. Matrix metalloproteases in BAL fluid of patients with cystic fibrosis and their modulation by treatment with dornase $\alpha$. Thorax 2002; 57: 930-934.

32 Gaggar A, Li Y, Weathington N, et al. Matrix metalloprotease-9 dysregulation in lower airway secretions of cystic fibrosis patients. Am J Physiol Lung Cell Mol Physiol 2007; 293: L96-L104. 